\title{
BMJ Open Phenotypic and antibiogram pattern of $V$. cholerae isolates from a tertiary care hospital in Mumbai during 2004-2013: a retrospective cross-sectional study
}

\author{
V Torane, ${ }^{1}$ S Kuyare, ${ }^{1}$ G Nataraj, ${ }^{1}$ P Mehta, ${ }^{1}$ S Dutta, ${ }^{2}$ B Sarkar ${ }^{2}$
}

To cite: Torane V, Kuyare S, Nataraj G, et al. Phenotypic and antibiogram pattern of $V$. cholerae isolates from a tertiary care hospital in Mumbai during 2004-2013: a retrospective cross-sectional study. BMJ Open 2016;6:e012638. doi:10.1136/bmjopen-2016012638

- Prepublication history for this paper is available online. To view these files please visit the journal online (http://dx.doi.org/10.1136/ bmjopen-2016-012638).

Received 25 May 2016 Revised 28 October 2016 Accepted 3 November 2016

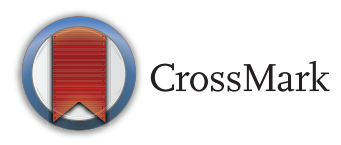

${ }^{1}$ Department of Microbiology, Seth G.S. Medical College \& KEM Hospital, Mumbai, Maharashtra, India

${ }^{2}$ Bacteriology Division, National Institute of Cholera and Enteric Diseases (NICED), Kolkata, West Bengal, India

Correspondence to Dr Vijaya Premchand Torane; vijayatorane@yahoo.co.in

\section{ABSTRACT}

Objectives: Cholera is a major gastroenteric disease with reports on fluctuation and resistance. Hence, the objective is to determine the trend in seasonality, resistance pattern, prevalent biotypes, serotypes and phage types between 2004 and 2013 among Vibrio cholerae isolates.

Design: A retrospective cross-sectional study. Settings: A single-centre study was carried out at a tertiary care hospital in a metropolitan city (Mumbai) of a developing country (India).

Methods: Records of stool specimen cultures of patients with suspected cholera from January 2004 to December 2013 were analysed. The organisms were identified as per standard protocol. Antimicrobial susceptibility testing was performed as per Clinical Laboratory Standard Institute. Biotyping, serotyping and phage typing were carried out. From the confirmed cases of cholera, demographic and laboratory details were noted. Descriptive analysis was used and the data were presented in the form of percentages.

Results: Vibrio cholerae was predominant in males and was isolated from $9.41 \%(439 / 4664)$ of stool specimens. Variability was found in terms of the gross appearance of stool specimens, seasonal trend and antibiotic resistance pattern. The antimicrobial susceptibility showed a waxing and waning pattern for most of the antibiotics (ampicillin, cefuroxime, chloramphenicol, tetracycline) tested, while for a few others the strains were either uniformly sensitive (gentamicin, norfloxacin) or resistant (trimethoprimsulfamethoxazole, nalidixic acid). All isolates belonged to subgroup 01 and biotype El Tor. The most common serotype was Ogawa. The predominant phage type was T2 (old scheme) and T27 (new scheme).

Conclusions: The predominant biotype, serotype and phage type were El Tor, Ogawa and T27 phage, respectively. The changing trends in antimicrobial resistance pattern over the years necessitate continued epidemiological and microbiological surveillance of the disease.

\section{Strengths and limitations of this study}

- Dissemination of such data is important for public health practices.

- Performance of biotyping, serotyping, antibiogram and phage typing.

- The data showed the changing trends over the years and the rising antimicrobial resistance which necessitate a continued surveillance study of cholera disease in Mumbai.

- This manuscript was prepared based only on the phenotypic pattern of Vibrio cholerae.

- The genotypic pattern and comparison between the two could not be carried out.

\section{INTRODUCTION}

Cholera is a major public health threat affecting the poor in developing countries ${ }^{1}$ and continues to spread across many countries. Historical records suggest that choleralike disease may have been first reported in the Era of Hippocrates (460-377 BC). ${ }^{2}$ In the year 2014, a total of 190549 cases of cholera were reported from 42 countries with a case fatality rate of $1.17 \%{ }^{1}$ During the same year in India, a total of 4031 cholera cases were reported from 12 different states, with 21 deaths. ${ }^{1}$ According to the International Health Regulations (2005), notification of all cases of cholera is no longer mandatory and this leads to underreporting, as observed by the National Institute of Cholera and Enteric Diseases (NICED), Kolkata, India. ${ }^{1}{ }^{3}$ The different causes of under-reporting could be a fear of negative impact on travel and trade in affected areas, limited surveillance of the disease, inconsistent case definitions and deficiency of laboratory diagnostic services. ${ }^{45}$ Integrated Disease Surveillance Project in 
India is a state-based surveillance system which reports cholera. ${ }^{6}$ However, reporting of cholera is still inadequate in India. ${ }^{7}$

The disease occurs throughout the year, the majority reported during the monsoon season. The causative agent, Vibrio cholerae (V. cholerae), has two biotypes, classical and El Tor. ${ }^{8}$ Each biotype has three serotypes: Ogawa, Inaba and Hikojima. ${ }^{9}{ }^{10}$ V. cholerae have demonstrated changes in prevalent biotypes and resistance over time. $^{11} 12$ The El Tor biotype (seventh pandemic) replaced the classical biotype causing the first six pandemics of cholera. Phage typing has contributed greatly to the understanding of the epidemiology of cholera. Phage typing at the NICED, Kolkata, India included the Basu and Mukherjee ${ }^{13}$ typing and new phage typing schemes. $^{14}$

The present study was carried out at a 2200 bedded tertiary care teaching hospital in the metropolitan city of Mumbai. As per the 2011 census, the population of Mumbai city was 124 million. ${ }^{15}$ Mumbai city is located on the western coast of Maharashtra along the banks of the Arabian sea and falls at latitude and longitude of $18^{\circ}$ $55^{\prime} \mathrm{N}$ and $72^{\circ} 54^{\prime} \mathrm{E}$, respectively. In Mumbai, 614 cases per thousand population are annually reported diarrhoeal disease burden. ${ }^{16}$ The microbiology department of our institute receives an average of 500 stool specimens annually from diarrhoeal cases for bacteriological study. The fluctuations in disease numbers and changing trends in resistance incited this study with an objective to determine trends in the seasonal association, resistance pattern, prevalent biotypes, serotypes and phage types of $V$. cholerae isolates over a period of 10 years.

\section{METHODS}

Procedure

A retrospective analysis of microbiology records of stool culture from January 2004 to December 2013 was carried out and the data were analysed on a yearly basis. Stool specimens were processed directly and after enrichment in alkaline peptone water as per standard protocol. ${ }^{17}$ The quality control of media and antibiotic discs used were carried out as per standard protocol. Specimens were inoculated on 5\% sheep blood agar, MacConkey's agar and Thiosulphate citrate bromothymol blue sucrose agar (HiMedia Laboratories Pvt, Mumbai). Specimens were also plated after enrichment in alkaline peptone water. Plates were incubated aerobically at $37^{\circ} \mathrm{C}$ and read after $24-48$ hours. Organisms were identified as per standard microbiological procedures. Non-lactose fermenting isolates which were Gram-negative motile bacilli, oxidase and catalase positive, fermenting glucose, sucrose and lactose (late) without gas, nitrate reducers, indole positive and citrate negative, lysine positive, arginine negative, ornithine positive, Cholera Red reaction positive and string test positive were presumptively identified as $V$. cholerae. The isolate identification was confirmed using $V$. cholerae polyvalent, Ogawa and Inaba antisera supplied by Becton Dickinson (Difco V.cholerae antiserum poly; Difco V.cholerae antiserum Ogawa; Difco $V$. cholerae antiserum Inaba). Biotyping was carried out as per standard phenotypic tests. Clinical and laboratory details of patients such as age, gender, residential address, date of specimen receipt, gross appearance of stool, reports of the hanging drop, culture and antibiotic sensitivity were noted. Repeat isolates from the same patient were excluded. Antimicrobial susceptibility test was carried out by the Kirby-Bauer disc diffusion method as per Clinical Laboratory Standard Institute. ${ }^{18}$ ATCC strain Escherichia coli 25922 was used for quality control of antimicrobial sensitivity testing. ${ }^{18}$ The following antibiotic discs (HiMedia Pvt, Mumbai) were tested-tetracycline $(30 \mu \mathrm{g})$, trimethoprim-sulfamethoxazole $(1.25 / 23.75)$, chloramphenicol $(30 \mu \mathrm{g})$, cefuroxime $(30 \mu \mathrm{g})$, ampicillin $(10 \mu \mathrm{g})$, gentamicin $(10 \mu \mathrm{g})$, furazolidone $(100 \mu \mathrm{g})$, norfloxacin $(10 \mu \mathrm{g})$ and nalidixic acid $(30 \mu \mathrm{g})$. The isolates were preserved in Brain Heart infusion agar with $1 \%$ sodium chloride. ${ }^{19}$ The preserved isolates were overlaid with sterile paraffin oil before transporting to NICED, Kolkata. Phage typing was carried out on 214 isolates by NICED, Kolkata from 2005 onwards using conventional the Basu and Mukherjee ${ }^{13}$ phage typing scheme and the New phage typing scheme. ${ }^{14}$ For the purpose of this study, a multidrug resistant (MDR) organism was defined as resistance to two or more classes of antimicrobial agents.

\section{Statistical analysis}

Descriptive analysis was used and presented in terms of percentage. The parameters analysed included age and gender distribution, residential address, month and year, gross findings of stool, results of the hanging drop, biotype, serotype, phage type, resistance pattern of the isolate and their changing trends.

\section{RESULTS}

\section{Demographics of the study participants}

About 4664 stool specimens of suspected cases were processed by microbiological culture during the study period. $V$. cholerae was isolated in 439 patients $(9.41 \%)$. Of the 439 patients, $313(71.3 \%)$ were adults and 126 $(28.7 \%)$ were children. The male to female ratio in adults was 2.6:1. In paediatric patients, the male to female ratio was 1.1:1. Considering the place of residence of patients infected with $V$. cholerae $(n=264)$, the majority $(71.2 \%)$ resided within a $5 \mathrm{~km}$ radius from the institution with no particular predominance of any area.

\section{Trend of V. cholerae cases and characteristics of stool specimens}

The cases were detected throughout the year, but peaked in the month of July for all the years and tapered from September onwards (table 1). A maximum number of cases $(n=79)$ were recorded in the year 2004, 
Table 1 Year-wise distribution of $V$. cholerae isolation

\begin{tabular}{|c|c|c|c|c|c|c|c|c|c|c|c|c|c|}
\hline Year & Jan & Feb & Mar & Apr & May & Jun & Jul & Aug & Sep & Oct & Nov & Dec & Total \\
\hline 2004 & 0 & 0 & 3 & 5 & 15 & 15 & 14 & 19 & 3 & 1 & 4 & 0 & 79 \\
\hline 2005 & 0 & 1 & 7 & 2 & 0 & 2 & 10 & 23 & 2 & 1 & 0 & 1 & 49 \\
\hline 2006 & 0 & 0 & 0 & 3 & 1 & 4 & 8 & 1 & 6 & 6 & 1 & 0 & 30 \\
\hline 2007 & 0 & 0 & 5 & 10 & 10 & 4 & 40 & $\mathrm{NC}$ & $\mathrm{NC}$ & $\mathrm{NC}$ & $\mathrm{NC}$ & $\mathrm{NC}$ & 69 \\
\hline 2008 & $\mathrm{NC}$ & $\mathrm{NC}$ & $\mathrm{NC}$ & $\mathrm{NC}$ & $\mathrm{NC}$ & 2 & 7 & 10 & 11 & 7 & 1 & 5 & 43 \\
\hline 2009 & 5 & 8 & 0 & 0 & 1 & 2 & 30 & 9 & 12 & 2 & 2 & 0 & 71 \\
\hline 2010 & 0 & 3 & 5 & 0 & 6 & 0 & 14 & 13 & 2 & 2 & 3 & 4 & 52 \\
\hline 2011 & 1 & 4 & 0 & 5 & 2 & 0 & 1 & 4 & 3 & 1 & 1 & 0 & 22 \\
\hline 2012 & 0 & 0 & 0 & 0 & 1 & 1 & 1 & 0 & 1 & 0 & 0 & 0 & 4 \\
\hline 2013 & 0 & 1 & 0 & 0 & 0 & 1 & 11 & 4 & 3 & 0 & 0 & 0 & 20 \\
\hline Total & 6 & 17 & 20 & 25 & 36 & 31 & 136 & 83 & 43 & 20 & 12 & 10 & 439 \\
\hline
\end{tabular}

NC, culture of stool specimens not performed; V. cholerae, Vibrio cholerae.

\begin{tabular}{lccc}
$\begin{array}{l}\text { Table } 2 \\
\text { cholerae }\end{array}$ & \multicolumn{4}{l}{ Year-wise distribution of different serotypes of $V}$. \\
\hline & Serotypes & & \\
Year & Ogawa & Inaba & Total \\
\hline 2004 & 79 & 0 & 79 \\
2005 & 47 & 2 & 49 \\
2006 & 11 & 19 & 30 \\
2007 & 69 & 0 & 69 \\
2008 & 40 & 3 & 43 \\
2009 & 68 & 3 & 71 \\
2010 & 47 & 5 & 52 \\
2011 & 22 & 0 & 22 \\
2012 & 4 & 0 & 4 \\
2013 & 20 & 0 & 20 \\
Total & 407 & 32 & 439 \\
\hline$V$.
\end{tabular}

V. cholerae, Vibrio cholerae.

followed by 2009 ( $n=71)$. Records of gross appearance were available in 368 stool specimens. Of this $77.4 \%$ (285) were rice watery. The remaining were green liquid $10 \%$ (37), yellow liquid $8.2 \%$ (30) or brown liquid specimens $3.8 \%$ (14). V. cholerae was also isolated from one specimen of green semisolid and reddish liquid stool.

\section{Biotypes and serotypes of $\mathrm{V}$. cholerae isolates}

Of the 439 isolates identified as $V$. cholerae, data for hanging drop were available from 327 specimens which were culture positive. Hanging drop was positive in $60.2 \%(\mathrm{n}=197)$ of culture-positive specimens. All isolates belonged to subgroup O1, biotype El Tor. The most common serotype was Ogawa $(407 ; 92.7 \%)$. No Inaba was isolated in 2004, 2007, 2011, 2012 and 2013 (table 2).

\section{Antimicrobial sensitivity pattern of the isolates}

More than $85 \%$ of the isolates were found to be sensitive to gentamicin and more than $90 \%$ to norfloxacin (table 3). On the other hand, $<7 \%$ were sensitive to nalidixic acid and $<27 \%$ to trimethoprim-sulfamethoxazole throughout the study period. For the other antimicrobials, varying trends were observed. The strains were maximally sensitive to tetracycline throughout the years except in $2008(51.2 \%)$. The sensitivity of $V$. cholerae to ampicillin reduced from $67.1 \%$ in 2004 to $46.2 \%$ in 2010 , and subsequently all strains were ampicillin resistant. Sensitivity to chloramphenicol increased from $0 \%$ in 2004 to $100 \%$ in 2007,2008 and 2009. It reduced to $20 \%$ in 2013. Susceptibility to cefuroxime increased from $34.2 \%$ in 2004 to $100 \%$ in 2008 and then decreased to $25 \%$ in 2012. Furazolidone sensitivity varied from $7.6 \%$ (2004) to $100 \%$ (2012). No interserotype difference in resistance pattern was noted. Throughout the decade, the treatment for cholera in our institute was doxycycline.

An analysis was also carried out to determine the changing trend in MDR (figure 1). Resistance to three or more than three antimicrobials tested was $96.2 \%$ in 2004, which decreased to $51.9 \%$ in 2010 . However, in 2013 , this increased to $90 \%$. Resistance to any two antimicrobials increased from $2.5 \%$ in 2004 to $43.7 \%$ in 2009 and then decreased to $10 \%$ in 2013 . Throughout the study period, only one isolate was susceptible to all the antimicrobials tested (2004).

\section{Phage types of $\mathrm{V}$. cholerae isolates}

A total of 214 strains of $V$. cholerae were sent for phage typing. The results of phage typing revealed Basu and Mukherjee type $\mathrm{T} 2$ to be the predominant phage $(84.6 \%)$. Non-typable strains (NT) accounted for $13.08 \%$ and Untypable strains (UT) accounted for $2.3 \%$. The New phage typing scheme showed T27 as the predominant phage $(77.6 \%)$, followed by T14 $(1.9 \%)$, T23 and T26 (1.4\%), T24 (0.9\%), T13, T20 and T25 $(0.46 \%)$. NT accounted for $13.08 \%$ and UT accounted for $2.3 \%$. No trend in phage type was observed.

\section{DISCUSSION}

In this study, $V$. cholerae was isolated from $9.41 \%$ of suspected cases, maximal during the monsoon season with El Tor, Ogawa and T27 being the most prevalent biotype, serotype and phage type, respectively. About one-third of cases $(28.7 \%)$ were reported of children. $V$. 
Table 3 Antimicrobial susceptibility patterns of $V$. cholerae isolates during 2004-2013 (\% susceptible)

\begin{tabular}{|c|c|c|c|c|c|c|c|c|c|}
\hline Year of isolation (n) & $\begin{array}{l}\text { TE } \\
\text { n (\%) }\end{array}$ & $\begin{array}{l}\mathrm{AM} \\
\mathrm{n}(\%)\end{array}$ & $\begin{array}{l}\mathrm{CH} \\
\mathrm{n}(\%)\end{array}$ & $\begin{array}{l}\text { GM } \\
\mathrm{n}(\%)\end{array}$ & $\begin{array}{l}\text { NF } \\
\mathrm{n}(\%)\end{array}$ & $\begin{array}{l}\text { co } \\
\text { n (\%) }\end{array}$ & $\begin{array}{l}\text { FZ } \\
\text { n (\%) }\end{array}$ & $\begin{array}{l}\text { NA } \\
\text { n (\%) }\end{array}$ & $\begin{array}{l}\text { CE } \\
\text { n (\%) }\end{array}$ \\
\hline 2004 (79) & 77 (97.5) & $53(67.1)$ & $1(0.0$ & 78 (98.7) & 75 (94.9) & 7 (8.9) & $6(7.6)$ & $3(3.8)$ & $27(34.2)$ \\
\hline 2005( & $42(85.7)$ & 19 (38.8) & $0(0)$ & 46 (93.9) & 45 (91.8) & $1(2)$ & $4(8.2)$ & $1(2)$ & $26(53.1)$ \\
\hline & & & & & & & & & \\
\hline & & & & & & & 12 & & 98.6) \\
\hline 2008 & $22(51.2)$ & $34(79.1)$ & $43 i$ & $42(97.7)$ & $42(97.7)$ & $6(14)$ & 7 (16.3) & $0(0)$ & $43(100)$ \\
\hline 2009 (71) & $59(83)$ & 25 (35.2) & $71(100)$ & 68 (95.8) & 69 (97.2) & 19 (26.8) & $18(25.4)$ & $5(7)$ & $68(95.8)$ \\
\hline 2010 (52) & 44 (84.6) & 24 (46.2) & 51 (98.1) & $52(100)$ & 49 (94.2) & $14(26.9)$ & 44 (84.6) & $3(5.8)$ & $38(73.1)$ \\
\hline & $22(100)$ & & 20 (90.9) & & & ל & & $0(0)$ & $22(100)$ \\
\hline & & & & & Do) & 0 & $4(100)$ & $0(0)$ & $1(25)$ \\
\hline $2013(20)$ & 19 (95) & $0(0)$ & $4(20)$ & $17(85)$ & $18(90)$ & $2(10)$ & $3(15)$ & $0(0)$ & $5(2$ \\
\hline
\end{tabular}

$\mathrm{AM}$, ampicillin; $\mathrm{CE}$, cefuroxime; $\mathrm{CH}$, chloramphenicol; $\mathrm{CO}$, trimethoprim-sulfamethoxazole; FZ, furazolidone; GM, gentamicin; NA, nalidixic acid; NF, norfloxacin; TE, tetracycline; V. cholerae, Vibrio cholerae.

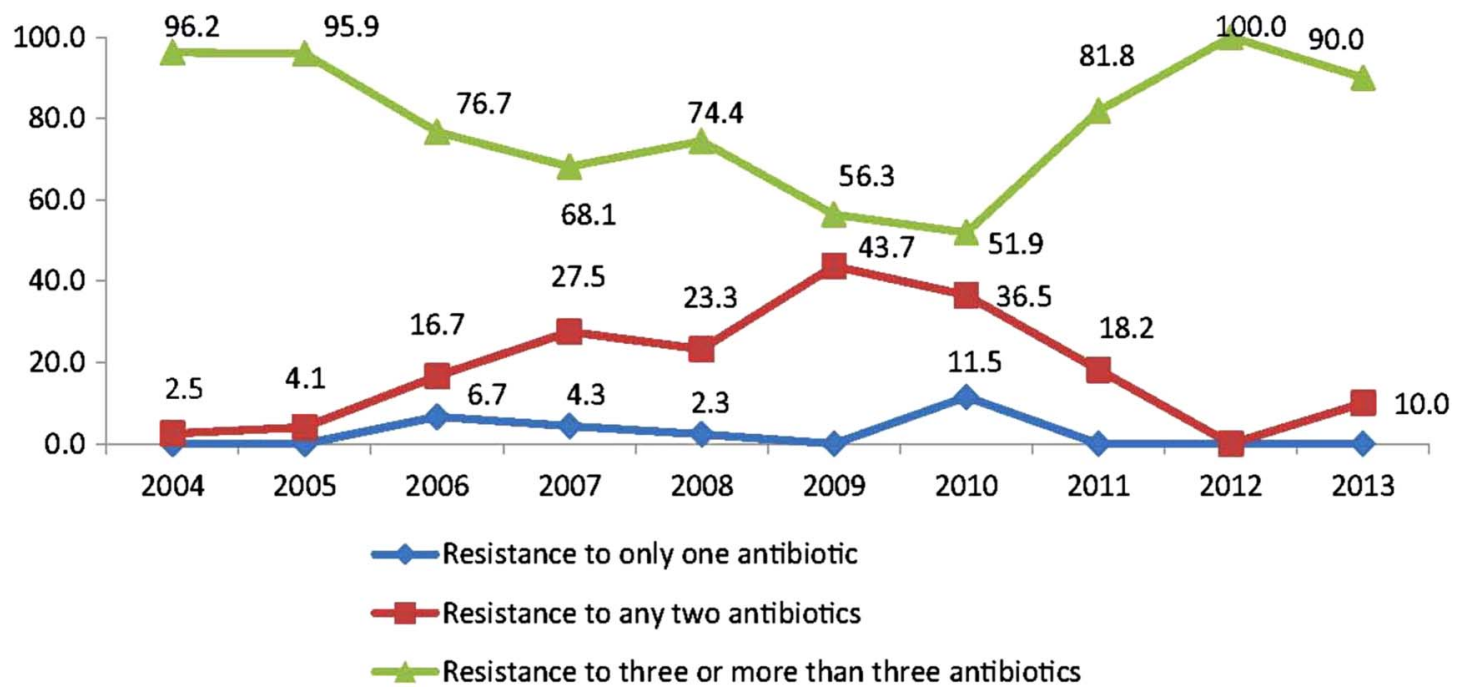

Figure 1 Changing trend in multidrug resistance pattern of $V$. cholerae (10 years). V. cholerae, Vibrio cholerae.

cholerae infection was common in males compared to females in the adult and paediatric population. ${ }^{20} 21$

A rising resistance was observed to most of the antimicrobials tested.

The isolation rate of $V$. cholerae from suspected cases was $9.41 \%$, which is similar to other studies. ${ }^{20} 2223$ Previous studies from Mumbai reported culture positivity rates ranging from $7.2 \%$ to $20 \% .{ }^{23}{ }^{24}$ Interestingly, V. cholerae was also isolated from stool specimens which were other than rice-watery. This may be due to the sample being collected during the initial infection period or due to mixed infection. Hence, irrespective of colour and consistency, the stool specimen should be cultured and investigated for $V$. cholerae.

The initial investigation on the stool specimen was hanging drop preparation. Our study showed that only $60.2 \%$ of culture positive specimens were positive for hanging drop. The reason for the high negativity rate may probably be attributed to the difficulty in differentiating between darting motility and actively motile organisms, delay in transport of specimens to the laboratory and effect of antibiotic therapy. Kulkarni et $a l^{25}$ have reported hanging drop positivity in $74 \%$ of culturepositive specimens.

A seasonal trend in the isolation of $V$. cholerae was seen. Cases initiated in February, peaked in July and tapered after September. This closely mimics the seasonal trends in monsoon. As per the data available from the India water portal organisation, the rainfall in Mumbai begins in June, peaks in July and gradually decreases in the month of August and by October it is negligible. ${ }^{26}$ The monsoon-related nature of disease coupled with the ability of $V$. cholerae to grow rapidly in warm humid environment and with Mumbai city receiving heavy showers with frequent flooding during monsoon, could be attributed to this seasonal trend of the disease..$^{22} 23$

Throughout the years, resistance to tetracycline was low $(<20 \%)$ except in $2008(49 \%)$. High-level tetracycline resistance was noticed by Kar et $a l^{27}$ from Odisha (2010), Bhattacharya et $a l^{28}$ from Kolkata (2007-2009), Kumar et $a l^{29}$ from Rayagada, Odisha (SeptemberOctober 2010), Borkakoty et $a \hat{l}^{30}$ from Assam (2007, 
2008 and 2010) and Taneja $e t a l^{31}$ from Chandigarh (2008). Plasmids are responsible for tetracycline resistance in $V$. cholerae. Vibrios do not stably carry plasmids, and hence the resistance pattern fluctuates. ${ }^{31}$ It is also possible that the irrational use of tetracycline or doxycycline in previous years could have led to the rapid emergence and spread of tetracycline-resistant isolates. ${ }^{31}$ Doxycycline is the drug of choice for the treatment of cholera in this institute and in the city. This is consistent with the susceptibility pattern over the years. Observing its continued therapeutic efficacy, it is recommended that the drug should be used judiciously for the management of acute diarrhoeal diseases in order to delay the development of resistance to the drug.

There was an increase in resistance to ampicillin in $2005(61 \%)$ with total resistance being observed during 2011-2013. Similar high rates of ampicillin resistance have been reported by Sharma et $a l^{32}$ from Delhi (2003 to 2005) and Palewar et $a l^{20}$ from Pune (2010 to 2012). One hundred per cent ampicillin resistance was also reported by Pal et $a l^{33}$ from the Eastern coast of Odisha (April-July 2009) and Kar et $a l^{27}$ from Odisha (2010).

Resistance to chloramphenicol was seen in spurts during 2004-2006, 2012 and 2013. For other years, sensitivity was more than $90 \%$. Goel and Jiang ${ }^{34}$ during 20042007, Pal et $a \ell^{35}$ during 2007 and Palewar et $a l^{20}$ during 2010-2012 reported 100\% sensitivity to chloramphenicol. Kar et $a l^{27}$ reported $100 \%$ resistance to chloramphenicol during 2010.Trimethoprim-sulfamethoxazole, furazolidone and nalidixic acid showed high levels of resistance similar to other studies. $^{20}{ }^{27} 7^{32-37}$ Trimethoprimsulfamethoxazole is the drug of choice in younger children. In view of the high level of trimethoprimsulfamethoxazole resistance and contraindication for use of tetracycline and fluoroquinolones in children, other options need to be considered. Low level of resistance was observed to norfloxacin $(<10 \%)$ and gentamicin $(<15 \%)$ throughout the study period. This finding is similar to other studies. ${ }^{20} 27323536$ A rising trend in MDR was observed over the years. A similar finding has been reported globally. ${ }^{37} 38$ The probable cause could be either due to spontaneous mutations or horizontal transfer of resistant genes within the gut flora and Vibrio species. ${ }^{37}$ The changing trend in antibiotic resistance over time may indicate the variability of treatment in the community.

The El Tor biotype of $V$. cholerae has completely replaced the classical biotype. The results of this study confirm these reports. ${ }^{38}$ Studies from India report the emergence of $V$. cholerae O139. ${ }^{39-42}$ However, in this study, all isolates were $V$. cholerae serogroup O1. The predominant serotype was Ogawa $(93.26 \%)$ with an occasional isolation of Inaba $(6.74 \%)$. In 2006, Inaba accounted for $63.3 \%$ of all isolates. This trend was similar to other studies. ${ }^{24} 36$ A periodic shift from Ogawa to Inaba has been reported either due to mutational changes in gene coding for serotype specificity ${ }^{43}$ or as a result of selection due to immune response during cholera infection ${ }^{44}$ or due to genetic reversal. ${ }^{45}$

The predominant phage type by the Basu and Mukherjee phage typing scheme was T2 (84.6\%). The new typing scheme showed T27 as the predominant phage type, which is similar to other studies. ${ }^{20} 2124253236$

\section{CONCLUSION}

El Tor Vibrios continue to be the predominant biotype, Ogawa the serotype and T27 the phage type. The changing trends over the years and the rising antimicrobial resistance necessitate continued epidemiological and microbiological surveillance for the disease.

Acknowledgements The authors are thankful to 'Accuwrite Medical Solutions' for copy editing the manuscript.

Contributors VT and SK were involved in the concept and design of the study while intellectual content, literature search, data acquisition, analysis and statistics were carried out by VT, SK and GN. Manuscript preparation was carried out by VT and SK while its editing and review was carried out by GN, $\mathrm{PM}, \mathrm{SD}$ and BS. SD and BS also carried out the phage typing procedure on isolates.

Funding This research received no specific grant from any funding agency in the public, commercial or not-for-profit sectors.

Competing interests None declared.

Ethics approval Ethical clearance was taken from the Institutional Ethics Committee (IEC/OA-97/2014) of Seth G S Medical College and King Edward Memorial Hospital, Mumbai, India.

Provenance and peer review Not commissioned; externally peer reviewed.

Data sharing statement No additional data are available.

Open Access This is an Open Access article distributed in accordance with the Creative Commons Attribution Non Commercial (CC BY-NC 4.0) license, which permits others to distribute, remix, adapt, build upon this work noncommercially, and license their derivative works on different terms, provided the original work is properly cited and the use is non-commercial. See: http:// creativecommons.org/licenses/by-nc/4.0/

\section{REFERENCES}

1. World Health Organisation. Cholera 2014. Wkly Epidemiol Rec 2015;90:517-44.

2. Blake PA. Historical perspectives on pandemic cholera. In: Wachsmuth IK, Blake PA, Olsvik O, eds. Vibrio cholerae and cholera. Molecular to global perspectives. Washington DC: American Society for Microbiology, 1994:293-6.

3. Sarkar BL, Kanungo S, Nair GB. How endemic is cholera in India? Indian J Med Res 2012;135:246-8.

4. Griffith DC, Kelly-Hope LA, Miller MA. Review of reported cholera outbreaks worldwide,1995-2005. Am J Trop Med Hyg 2006;75:973-7.

5. Zuckerman JN, Rombo L, Fisch A. The true burden and risk of cholera: implications for prevention and control. Lancet Infect Dis 2007;7:521-30.

6. Training manual for medical officers for Hospital based disease surveillance. Integrated Disease Surveillance Project by National Centre for Disease Control. http://idsp.nic.in/WriteReadData/OldSite/ usermanaul/manual_for_MO.pdf (accessed 24 Oct 2016).

7. Kanungo S, Sah BK, Lopez AL, et al. Cholera in India: an analysis of reports, 1997-2006. Bull World Health Organ 2010;88:185-91.

8. Gardner AD, Venkatraman KV. The antigens of the cholera group of vibrios. J Hyg (London) 1935;35:262-82.

9. Kabeshima T. Immunological properties of the cholera bacillus. $C R$ Soc Biol 1918;81:618.

10. Nobechi K. Contributions to the Knowledge of Vibrio cholerae. 3 Immunological Studies on the Types of Vibrio cholerae. Scientific 
Reports. Vol 2. Government Institute for Infectious Diseases, Tokyo Imperial University, 1923:1-87.

11. Faruque AS, Alam K, Malek MA, et al. Emergence of multidrug-resistant strain of Vibrio cholerae $\mathrm{O} 1$ in Bangladesh and reversal of their susceptibility to tetracycline after two years. $J$ Health Popul Nutr 2007;25:241-3.

12. Safa A, Nair GB, Kong RY. Evolution of new variants of Vibrio cholerae O1. Trends Microbiol 2010;18:46-54.

13. Basu S, Mukherjee S. Bacteriophage typing of Vibrio El Tor. Experientia 1968;24:299-300.

14. Chattopadhyay DJ, Sarkar BL, Ansari MQ, et al. New phage typing scheme for Vibrio cholerae O1 biotype El Tor strains. J Clin Microbiol 1993;31:1579-85.

15. Mumbai city census data. http://www.census2011.co.in/census/city/ 365-mumbai.html (accessed Apr'16)

16. Kumar Karn S, Harada H. Field survey on water supply, sanitation and associated health impacts in urban poor communities-a case from Mumbai City, India. Water Sci Technol 2002;46:269-75.

17. Scott AC. Laboratory control of antimicrobial therapy. In: Colle JG, Duguid JP, Fraser AG, et al, eds. Mackie and McCartney practical medical microbiology. 13th edn. Vol 2. Edinburgh: Churchill Livingstone, 1989:161-81.

18. Clinical Laboratory Standard Institute (CLSI). Performance standards for Antimicrobial Susceptibility Testing. Informational supplement as applicable for the years 2004-2013. Fourteenth to Twenty-Third Informational Supplement. CLSI document M100-S14 to M100-S23. Wayne, PA: Clinical and Laboratory Standards Institute, 2004-2013.

19. Centre for Disease Control and Prevention. Laboratory methods for the diagnosis of epidemic dysentery and cholera. 1999. http:// www.cdc.gov/cholera/pdf/Laboratory-Methods-for-the-Diagnosisof-Epidemic-Dysentery-and-Cholera.pdf (accessed Apr' 16).

20. Palewar MS, Choure AC, Mudshingkar S, et al. Typing and antibiogram of Vibrio cholerae isolates from a Tertiary Care Hospital in Pune: a 3 year study. J Glob Infect Dis 2015;7:35-6.

21. Chander J, Kaistha N, Gupta V, et al. Epidemiology and antibiogram of Vibrio cholerae isolates from a tertiary care hospital in Chandigarh, North India. Indian J Med Res 2009;129:613-7.

22. Mohanty S, Kapil A, Das BK. Seasonality and antimicrobial resistance pattern of Vibrio cholerae in a tertiary care hospital of North India. Trop Doct 2004;34:249-51.

23. Mathur M, De A, Saraswathi K, et al. Vibrionaceae from cases of acute diarrhoea and their antimicrobial sensitivity pattern-a five year prospective study. Indian J Med Microbiol 2003;21:199-201.

24. Turbadkar SD, Ghadge DP, Patil S, et al. Circulating Phage type of Vibrio cholerae in Mumbai. Indian J Med Microbiol 2007:25:177-8.

25. Kulkarni RD, Patil SA, Kulkarni VA, et al. An outbreak of cholera in the Sangli District of Maharashtra. Indian J Med Microbiol 2007;25:76-8.

26. Data of Rainfall in Mumbai city. http://www.indiawaterportal.org/sites/ indiawaterportal.org/files/imd_district-wise_rainfalldata_2004-2010. xls (accessed Apr'16)

27. Kar SK, Pal BB, Khuntia HK, et al. Emergence and spread of Tetracycline resistant Vibrio cholerae O1 El Tor variant during 2010 cholera epidemic in the tribal areas of Odisha, India. Int $J$ Infect Dis 2015;33:45-9.
28. Bhattacharya $\mathrm{K}$, Kanugo S, Sur D, et al. Tetracycline resistant Vibrio cholerae O1, Kolkata, India. Emerging Infect Dis 2011:17:568-9.

29. Kumar P, Jain M, Goel AK, et al. Tetracycline resistant $V$. cholerae O1 biotype El Tor serotype Ogawa with classical ctx B from a recent cholera outbreak in Orissa, Eastern India. J Infect Public Health 2012;5:217-19.

30. Borkakoty B, Biswas D, Devi U, et al. Emergence of classical ctx B genotype 1 and tetracycline resistant strains of Vibrio cholerae O1 El Tor in Assam, India. Trans R Soc Trop Med Hyg 2012;106:382-6

31. Taneja N, Samanta P, Mishra A, et al. Emergence of tetracycline resistance in Vibrio cholerae O1 biotype El Tor serotype Ogawa from north India. Indian J Pathol Microbiol 2010;53:865-6.

32. Sharma NC, Mandal PK, Dhillon R, et al. Changing profile of Vibrio cholerae 01, O139 in Delhi and its periphery (2003-2005). Indian J Med Res 2007;125:633-40.

33. Pal BB, Khuntia HK, Samal SK, et al. Large outbreak of cholera caused by El Tor variant Vibrio cholerae O1 in the eastern coast of Odisha, India during 2009. Epidemiol Infect 2013;141:2560-7.

34. Goel AK, Jiang SC. Genetic determinants of virulence, antibiogram and altered biotype among the Vibrio cholerae $\mathrm{O} 1$ isolates from different cholera outbreaks in India. Infect Genet Evol 2010;10:815-19.

35. Pal B, Khuntia H, Samal S, et al. Epidemics of severe cholera caused by El Tor Vibrio cholerae O1 Ogawa possessing the ctxB gene of the classical biotype in Orissa, India. Int $J$ Infect Dis 2010;14:384-9.

36. De A, Mathur M. Isolation of Vibrio cholera El Tor Serotype Inaba in 2006 and the most common Phage type of $V$. cholerae in Mumbai. Indian J Community Med 2009;34:78.

37. ThapaShrestha U, Adhikari N, Maharjan R, et al. Multidrug resistant Vibrio cholerae $\mathrm{O} 1$ from clinical and environmental samples in Kathmandu city. BMC Infec Dis 2015;15:104.

38. Mahapatra T, Mahapatra S, Babu GR, et al. Cholera outbreaks in South and Southeast Asia: descriptive analysis, 2003-2012. Jpn J Infect Dis 2014;67:145-56.

39. Ramamurthy T, Garg S, Sharma R, et al. Emergence of novel strains of Vibrio cholerae with epidemic potential in South and Eastern India. Lancet 1993;341:703-4.

40. Nair G, Albert M, Shimada T, et al. Vibrio cholerae 0139 Bengal: the new serogroup causing cholera. Rev Med Microbiol 1996;7: 43-52.

41. Narang P, Mendiratta DK, Deotale VS, et al. Changing patterns of Vibrio cholerae in Sevagram between 1990 and 2005. Indian J Med Microbiol 2008;26:40-4

42. Sundaram SP, Revathi J, Sarkar BL, et al. Bacteriological profile of cholera in Tamil Nadu (1980-2001). Indian J Med Res 2002;116:258-63.

43. Bhaskaran $\mathrm{K}$, Gorill $\mathrm{RH}$. A Study of antigenic variation in Vibrio cholerae. J Gen Microbiol 1957;16:721-9.

44. Nobechi K, Nakano E. Studies on shifting of the serotypes of cholera vibrios. The first report: studies in vitro. Symposium on Cholera, Palo Alto, Calif. Bethesda: National Institute of Health, 1967:119-21.

45. Sack RB, Miller CE. Progressive changes of Vibrio serotypes in germ free mice infected with Vibrio cholerae. J Bacteriol 1969;99:688-95. 